\title{
Research on Crowdsourcing Self-organization Formation Mechanism Based on Entropy Model Interpretation
}

\author{
Gao Xirong*, Yang Fei \\ School of Economics and Management, Chongqing University of Posts and Telecommunications \\ Chongqing, 400065
}

\begin{abstract}
In order to improve the crowdsourcing operation efficiency, the paper proposes the concept of crowdsourcing selforganization and introduces its connotation and characteristics systematically. By analyzing the logical relationship between entropy and crowdsourcing self-organization, the entropy model of crowdsourcing self-organization is constructed and the condition variables of the crowdsourcing self-organization formation are explored deeply, respectively including: crowd target task space, crowd acceptance probability and crowdsourcing environment entropy change. The research enlightenment can promote the formation of crowdsourcing selforganization by "paying attention to the internal needs of the public, establishing the interest-oriented crowdsourcing mode", "enhancing the crowd talents and enhancing crowd acceptance confidence" as well as "improving the external environment of crowdsourcing and increasing the crowd participation enthusiasm", etc.
\end{abstract}

Keywords-Crowdsourcing; Self-organization; Formation mechanism; Entropy model

\section{INTRODUCTION}

The term crowdsourcing is originated in the United States, which was first proposed by Jeff Howe, a journalist for Wired Magazine in the United States. The so-called "crowdsourcing" means: a kind of behavior that an enterprise or an organization outsources the internal work tasks which should be performed by its employees to non-specific group and crowd who are voluntarily willing to undertake the tasks in order to reduce its operating costs ${ }^{[1]}$. In essence, crowdsourcing is an open crowd innovative behavior with the advantages of "smartly using collective intelligence", "inspiring users to create content", "reducing corporate costs", and "enhancing the task resolution efficiency". However, in the actual crowdsourcing call process, it is not difficult to find that the crowd's enthusiasm for participation in crowdsourcing activities is not high, which greatly affects the role of crowdsourcing in promoting innovation performance. In order to improve the operating efficiency of crowdsourcing, it is imperative to study how to enable the crowd to actively participate in crowdsourcing activities, increase the crowd's enthusiasm for participation in crowdsourcing activities, thereby promoting the formation of more orderly and more efficient crowdsourcing self- organization, that is, a research on crowdsourcing selforganization formation mechanism.

\section{THEORETICAL BASIS}

\section{A. The concept and characteristics of crowdsourcing self- organization}

At present, there are fewer relevant researches on "crowdsourcing self-organization" in the academic community. In China National Knowledge Infrastructure, only one article can be retrieved with the theme of "crowdsourcing selforganization". There are few existing researches systematically defining the concept of crowdsourcing self-organization. From the perspective of thermodynamics, self-organization means a process of improving the ordering degree of the system by continuously reducing the entropy content of its own system under the influence of internal mechanisms and the external environment ${ }^{[3]}$. After combining the above definitions of "crowdsourcing" and "self-organization", the crowdsourcing self-organization is defined as follows. The so-called "crowdsourcing self-organization" means: an orderly structure with similar organization effects formed that the non-specific crowd without organized relationships are irrelevant subjectively, but they can solve the crowdsourcing tasks under a certain rule of tacit agreement. In Short, crowdsourcing selforganization means an organized group structure formed that non-specific crowd are voluntarily willing to participate in the crowdsourcing tasks they are interested in

Crowdsourcing self-organization is characterized by nonmandatory, non-hierarchical, similarly organizational, and evolutionary properties. Among them, non-mandatory property means that non-specific crowd have complete personal selection right for whether to participate in crowdsourcing activities or what kinds of crowdsourcing tasks they will choose, and this right will not be forced by others or the environment, but is the most important characteristic of crowdsourcing self-organization; The non-hierarchical property means that there is no a kind of organization relationship (i.e. power jurisdiction or hierarchical stratification) among nonspecific crowd, but a non-organizational relationship that everyone can make decisions autonomously and be responsible for their own decisions with the crowdsourcing task as the center; The similarly organizational property emphasizes the 
effectiveness of crowdsourcing self-organization achieved in solving crowdsourcing tasks that are similar to organized behaviors, that is, a large number of crowdsourcing tasks can be solved within a certain period of time; The evolutionary property means that the number of tasks solved through the crowdsourcing mode and the solving efficiency have been significantly improved with the gradual improvement of the crowdsourcing environment and the gradual increase in the crowdsourcing ability.

\section{B. The logical relationship between crowdsourcing self- organization formation and entropy}

According to the above definition of crowdsourcing selforganization, we can easily define the concept of "crowdsourcing self-organization formation". Crowdsourcing self-organization formation means a process of transforming a disorderly structure that the social crowd are dispersed and distributed like loose sand in the crowdsourcing selforganization to an orderly structure that there are both cooperative and competitive relationships based on common interests. From the research perspective of thermodynamics and system theory, the process of transforming a disorderly structure of crowdsourcing self-organization to an orderly structure is always accompanied by changes in entropy. Entropy is a parameter that indicates the state of matter in thermodynamics, and each entropy value corresponds to its state of matter ${ }^{[4]}$. The change in entropy value (i.e., the change in entropy) often means a change in the state of matter, which just also have corresponding coherence points with the crowdsourcing self-organization formation. If entropy can be used to represent the structure state of crowdsourcing selforganization, it will be conducive to exploring the key path in the process of crowdsourcing self-organization, thereby from the key path; the formation mechanism of crowdsourcing selforganization can be researched from the perspective of dynamic change.

\section{CROWDSOURCING SELF-ORGANIZATION ENTROPY MODEL CONSTRUCTION}

\section{A. Modeling ideas}

The goal of the text is to explore the formation mechanism of crowdsourcing self-organization, that is, to study the evolution process mechanism of crowdsourcing selforganization from disorderly structure to orderly structure, and therefore, it is necessary to measure the orderly structure of crowdsourcing self-organization. Since entropy can objectively measure the disorder degree of the system, changes in entropy value can be used to reflect changes in the disorder degree of crowdsourcing self-organization. Therefore, it plans to construct a crowdsourcing self-organization entropy model based on the principle of entropy change and explore the key path of decreasing the entropy value of crowdsourcing selforganization (that is, increasing the degree of order) so as to better explain the formation mechanism of crowdsourcing selforganization.

\section{B. Model building}

According to the explanation of entropy in thermodynamic theory, it can be easily known that: Entropy is a measure of the disorder degree of system, and the greater the system entropy is the more disorderly the system will be ${ }^{[5]}$. The research goal of the text is to achieve the formation of crowdsourcing selforganization, even if the final state of the crowdsourcing selforganization system is a very orderly structure, as long as the total entropy value of the crowdsourcing self-organization system is gradually reduced, the purpose of transforming "crowdsourcing self-organization from disorderly structure to orderly structure" can be achieved. Therefore, before exploring how to reduce the total entropy value of crowdsourcing selforganization system, a crowdsourcing self-organization entropy model should be built firstly to represent the total entropy of crowdsourcing self-organization system. The model building steps are as follows.

Assuming that $c=\left\{c_{1}, c_{2}, \cdots, c_{m}\right\}$ is the crowd set participating in the crowdsourcing in the crowdsourcing selforganization system, among which $c_{i}(1 \leq i \leq m)$ indicates any one of crowd individual in the crowdsourcing selforganization system; $d=\left\{d_{1}, d_{2}, \cdots, d_{n}\right\}$ is a set of published tasks in the crowdsourcing self-organization system, among which $d_{j}(1 \leq j \leq n)$ indicates any one of crowdsourcing tasks in the crowdsourcing self-organization

system, $H$ is the system entropy. The entropy of any one $c_{i}$ of crowd in a crowdsourcing self-organization system can be expressed as:

$$
H\left(c_{i}, d\right)=-\sum_{j=1}^{k_{i}} p_{i j} \ln p_{i j}
$$

In formula (1), $p_{i j}$ indicates the probability that the crowd $c_{i}$ participate in the task $d_{j}$, and $k_{i}$ indicates the target task space of the crowd $c_{i}$, among which $k_{i}$ satisfies $0<k_{i} \leq n$ and is an integer.

Since the entropies of the crowdsourcing self-organization system contains all crowd entropies, all crowd entropies in the system are as follows:

$$
H(c, d)=-\sum_{i=1}^{m} H\left(c_{i}, d\right)=-\sum_{i=1}^{m} \sum_{j=1}^{k_{i}} p_{i j} \ln p_{i j}
$$

In formula (2), $m$ indicates the total number of crowd in the system, and all crowd entropies $H(c, d)$ in the system can be obtained from the sum of the entropies of the crowd individuals.

In addition, since crowdsourcing self-organization relies on the crowdsourcing platform, the degree of order of crowdsourcing self-organization will also be affected by the external environment of the crowdsourcing platform, which 
can be understood as follows: the crowdsourcing platform external environment will change the entropy value of the crowdsourcing organization system. Therefore, the entropy change from the exchange of material and energy between the crowdsourcing self-organization system and the external environment should also be considered, which can be directly expressed by $\Delta S_{e}$ to prevent the model from becoming too complicated.

In summary, the total entropy model of crowdsourcing selforganization is shown in formula (3):

$$
H\left(c, d, \Delta S_{e}\right)=H(c, d)+\Delta S_{e}
$$

\section{Model analysis}

By analyzing the above crowdsourcing self-organization entropy model, we have explored the path variables that reduce the value of crowdsourcing self-organization entropy (that is, increase the order degree), respectably including target task space variable $k$, the probability $p$ of crowd participation in crowdsourcing tasks and the entropy change $\Delta S_{e}$ from the external environment. Therefore, we have three paths which can reduce the crowdsourcing self-organization entropy value. Path $\mathrm{I}$ is to narrow the crowd target task space $k$. It can be known from the mathematical induction that the entropy $H$ of any one of crowd will be increased with the increase in the crowd target task space $k$, and the value $k$ should be reduced in order to reduce the total entropy. Path II is to polarize the crowd acceptance probability $p$.After mathematical derivation it can be known that when the probabilities for the crowd $c_{i}$ in choosing any target task are equal, i.e. $p_{i 1}=p_{i 2}=\cdots=p_{i k_{i}}=1 / k_{i}$, the entropy of the crowd $c_{i}$ should be taken as the maximum value, and when the probability for the crowd $c_{i}$ in choosing a certain task $d_{j}$ is 1 and the choosing probabilities of the rest tasks are taken as 0 , the crowd entropy $H$ should be the minimum value, and therefore, the process of decreasing the entropy value is the polarization process of the probability $p$ of crowd acceptance. Path III is to introduce the crowdsourcing external environment
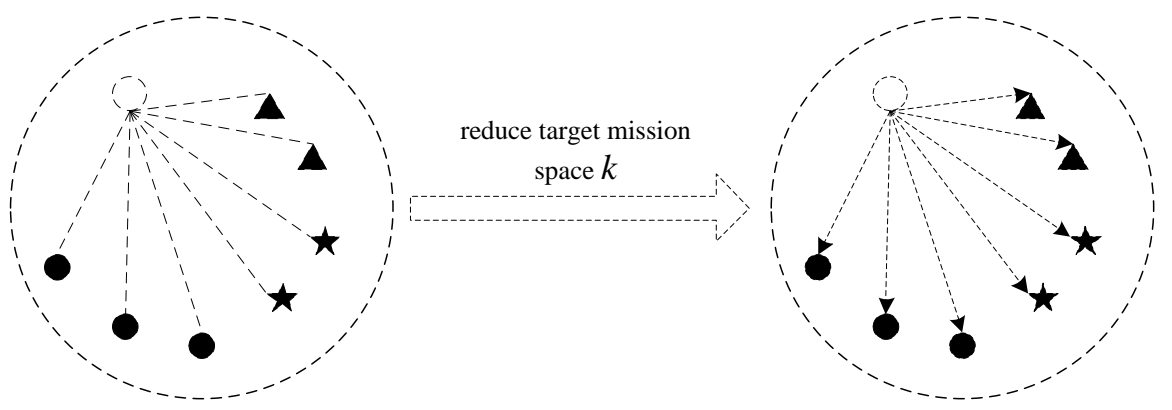

the crowd

$\downarrow \Delta$ different types of crowdsourcing tasks

willing to participate in the task not willing to participate in the task

Fig. 1 Process of crowd target task space reduction 


\section{B. The polarization process of the crowd acceptance probability}

Similarly, according to the crowdsourcing self-organization entropy model, it can be known that when the crowd accepts the probabilities $p$ of all possible crowdsourcing tasks are equal, the total entropy of the system $H$ should be the maximum value. Therefore, the achievement of the imbalanced and polarized probability $p$ of crowd participation in crowdsourcing tasks in crowdsourcing self-organization systems can decrease the entropy $H$ of crowdsourcing selforganization system. It means that the probability of a crowd individual participating in a task approaches 1 and the probability of participating in other tasks approaches 0 , that is, the crowd have decided which task they will participate in a
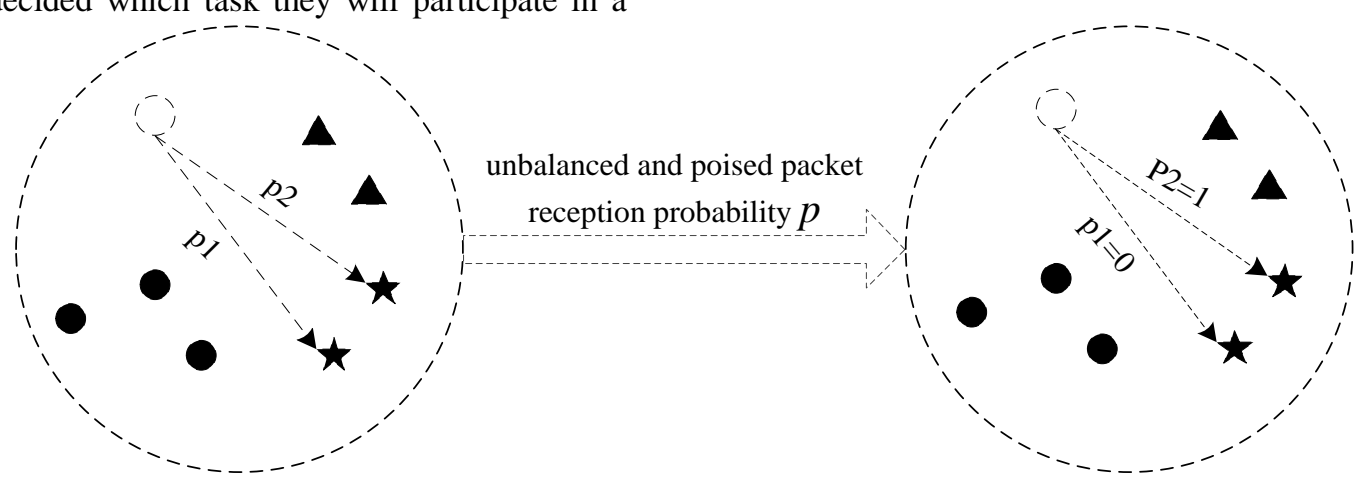

Fig. 2 Process of polarizing the acceptance probability of the crowd

\section{The introduction process of the crowdsourcing environment negative entropy}

According to the crowdsourcing self-organization entropy model, it can be known that when the crowd interacts with the outside world and the entropy flow $\Delta S_{e}$ introduced is negative, the system entropy of crowdsourcing self-organization can be reduced. In terms of theory, the introduction of negative entropy in the process of interacting with the outside world can improve the external environment of crowdsourcing platforms, such as improving the usability of crowdsourcing platform and strengthening the crowdsourcing incentive mechanism, etc., because the crowd cannot develop the corresponding crowdsourcing activities after leaving the crowdsourcing platform. Therefore, for the crowd, the quality of the certain type of task group (to prevent the model from being too complicated, assuming that every crowd only participates in one task at a time). Therefore, by polarizing the probability of crowd acceptance by some measures, the crowd can successfully participate in the crowdsourcing tasks they are interested in and accelerate the formation of crowdsourcing self-organization. Figure 2 describes the process of polarizing the acceptance probability of the crowd joining the crowdsourcing platform. 


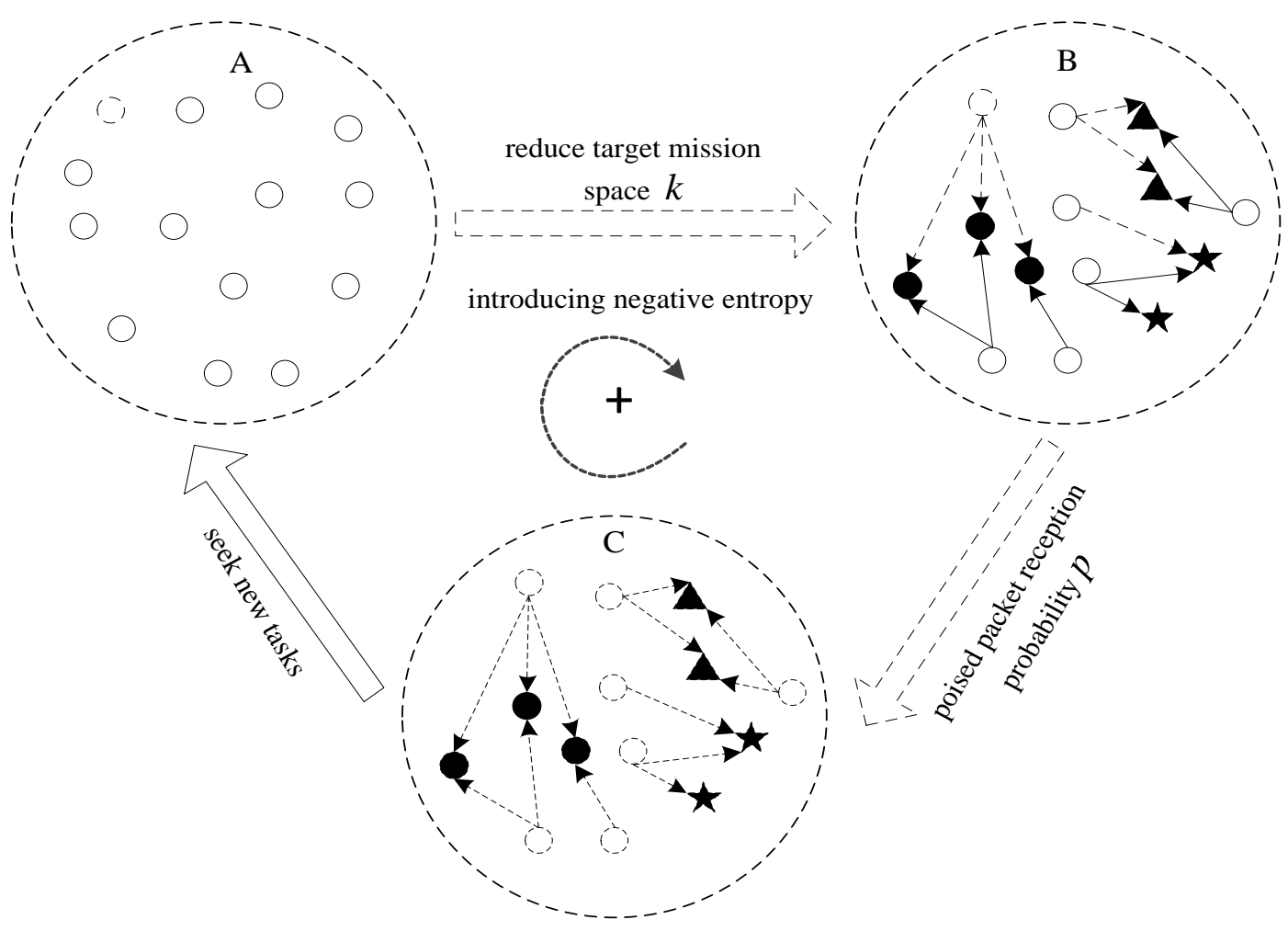

Fig. 3 Process of crowdsourcing self-organization formation

Figure 3 portrays a complete process from task releasing and gathering start to task solution, respective discretion and next task start for the crowd with task as the condensation nucleus. It contains the three states represented by the crowd. State A is the initial state, which represents the chaotic movement with no goal when the crowd enter into the crowdsourcing platform. State B is the middle state, which represents the low-order movement when the crowd have found the target task space. State $\mathrm{C}$ is the result state, which represents the high-order movements that the crowd have determined the final target task and begun to solve the task. It is precisely because the crowd reduces the target task space, State A will be transformed to State B, then the development of State B to State $\mathrm{C}$ will be achieved through polarizing the probability of the crowd participation in the task, finally, the temporary property of the crowdsourcing task will prompt the disintegration of the crowd and the starting of finding the next task, and however, the introduction of external negative entropy flows can protect the entire process of crowdsourcing self-organization formation. With the cycle of task solving, the orderly and efficient crowdsourcing self-organization formation will be gradually formed.

\section{The Mechanism Analysis of CROWdSOURCING SelF- ORGANIZATION PROCESS}

By analyzing the process of crowdsourcing selforganization formation, we can disassemble it into three stages: reducing the task space, polarizing the acceptance probability and entering into a new cycle, and then discuss how to achieve each stage of crowdsourcing self-organization formation.

At the first stage, the crowd will be transformed from the disorderly initial state A to the low-order middle state B, i.e., the crowd target task space will be reduced, this process is mainly affected by the internal needs of the crowd. When the crowd understand their own leading needs and determine the types of tasks they are interested in, the purpose of narrowing the target task space will have been achieved. In fact, it includes introducing negative entropy, helping the crowd clear the type of individual goals and tasks through the improvement of the external environment, reducing their search costs (for example, the clear classification of many tasks can facilitate the rapid positioning of the types of tasks for the crowd) or using the big data technology to establish the task recommendation mechanism, deeply excavating the internal needs of the crowd, and predicting and recommending the corresponding crowdsourcing tasks according to their behavior performance.

At the second stage, the crowd will be transformed from the low-order intermediate state B to the high-order result state C, i.e., the probability of the crowd acceptance will be polarized, this process is mainly affected by the ability of the crowd. 
After the crowd determine the types of tasks, the appropriate task will be chosen based on the actual ability of the individual and then its solving will be started. The above process includes two levels, one is to correctly assess the ability and determine the finally participated crowdsourcing tasks, and the other is to begin to formulate the solutions based on the chosen task requirements. The former requires a clear description of crowdsourcing tasks so that the crowd can reasonably match their own abilities and crowdsourcing tasks. The latter requires the crowd to have the ability to solve tasks. Only when the crowd have solved the crowdsourcing tasks, the effect of crowdsourcing self-organization can be achieved, and therefore, the technical support or training services, etc. can be provided in order to improve public crowdsourcing capabilities, both of which can guarantee the effectiveness of crowdsourcing jointly.

At the third stage, the crowd will be transformed from the high-order result state $\mathrm{C}$ to the disorderly initial state $\mathrm{A}$, i.e., the crowd after completing the crowdsourcing task will be dissolved, and then it will begin to search for new target task spaces, and this process is mainly affected by the crowdsourcing environment. The quality of the crowdsourcing environment directly affects the experience of crowdsourcing activities, and the quality of the experience directly affects the continuous participation behavior of the crowd, i.e., whether it will continue to search for new crowdsourcing tasks. The crowdsourcing experience consists of the following five parts: operability, interactivity, authenticity, fairness and protection. Operability means the ease of use of crowdsourcing platforms by the crowd; Interactivity means the convenience degree of communication of the crowd with the platform or others; Authenticity means the true degree of crowdsourcing task information in the platform; Fairness means the degree of fairness of the task solution selection means and results; Protection means the degree of protection of the property rights for crowd creative ideas by the platform. From the above five aspects, the crowdsourcing environment can be improved, the crowd experience can be enhanced, the continuous participation of the crowd can be encouraged, and the crowdsourcing self-organization can be eventually formed.

\section{CONCLUSION}

The concept of crowdsourcing self-organization is herein innovatively proposed, and the formation mechanism of crowdsourcing self-organization is researched by using the model analysis method through the construction of crowdsourcing self-organization entropy model. The conclusions of the research are as follows: (1) Crowdsourcing self-organization means an orderly structure with similar organization effects formed that the non-specific crowd without organized relationships are irrelevant subjectively, but they can solve the crowdsourcing tasks under a certain rule of tacit agreement; (2) The crowdsourcing self-organization is characterized by non-mandatory, non-hierarchical, similarly organizational, and evolutionary properties. Non-mandatory property is the most important characteristic of crowdsourcing self-organization; (3) The formation path of the selforganization includes reducing the crowd target task space, increasing the probability of crowd acceptance and increasing the negative entropy of crowdsourcing; (4) From the micro level, the formation process of crowdsourcing self-organization is divided into three stages, which correspond to the initial stage of crowd's non-targeted and chaotic movements, the middle stage of "the crowd finding the target task space and their low-order movements" and the final stage of "the crowd determining the final target task and beginning to resolve the task and their high-order movements"; (5) From the macro level, the formation of crowdsourcing self-organization is a positive cycle process in which crowdsourcing tasks can be resolved one by one. With the cycle of task resolution, the crowdsourcing self-organization will run more orderly and the solving efficiency of crowdsourcing tasks will become higher. In addition, in terms of promoting the formation of crowdsourcing self-organization, the three recommendations are given herein: (1) Pay attention to the internal needs of the crowd and establish an interest-oriented crowdsourcing operation mechanism; (2) Promote the crowd crowdsourcing ability and enhance the crowd's confidence in the acceptance; (3) Improve the crowdsourcing external environment and increase the crowd participation in crowdsourcing activities.

\section{REFERENCES}

[1] Howe J. The rise of crowdsourcing [J]. Wired Magazine, 2006, 14(6): 176-183.

[2] Wang Shu, Chen Jin and Liang Jing. Analysis of Collaborative SelfOrganization Innovation Effects of Network Crowdsourcing Model [J]. Science Research Management, 2014, 35 (04):26-33.

[3] Zhang Zhifeng, Xiao Renbin and Liu Meiling. Enterprise System Entropy Change Model Based on Dissipative Structure [J]. Industrial Engineering \& Management, 2007(01):15-19.

[4] Bai Jingkun, Wang Jian and Zhang Zhenzhen. Research on the Formation Mechanism of Platform Enterprise Network SelfOrganization -Taking Taobao as an Example [J]. China Soft Science, 2017(05):171-180.

[5] Shen Qian, Huang Yuan, Ma Ning and Liu Yijun. Research on "Entropy Reduction Points" in the Evolution of Complex Networks: Taking the Evolution of Microblog Communication Network as an Example [J]. Journal of Mathematics in Practice and Theory, 2015, 45(03): 282-290. 